\title{
In Situ Transformation of Two Dimensional MoS2 from Amorphous Precursor
}

\author{
Peter Tieu, Wenpei Gao and Xiaoqing Pan
}

${ }^{1}$ University of California-Irvine, Irvine, California, United States

Two dimensional transition metal dichalcogenides such as $\mathrm{MoS}_{2}$ and $\mathrm{WS}_{2}$ have multiple applications in the field of electronics, catalysis, sensors, and energy storage. In particular, molybdenum disulfide has been heavily used in the bulk form for centuries as a solid lubricant and the formation of the two dimensional variant has been investigated through multiple means such as X-ray diffraction and X-ray absorption spectroscopy in conjunction with theoretical modelling. [1] Thermolysis of $\left(\mathrm{NH}_{4}\right)_{2} \mathrm{MoS}_{4}$ in an inert atmosphere is the most heavily studied transformation due to the ease of formation and scalability. Understanding the transformation process through the intermediate stages is essential for controlling the formation of the two dimensional material. Here, we observe the formation of two dimensional $\mathrm{MoS}_{2}$ solely from electron beam irradiation in an aberration corrected STEM at atomic resolution, layer formation was tracked. A power spectrum analysis is applied to the fast Fourier transformation of the images to analyze the dynamics of the bonding changes during the transformation.

$\left(\mathrm{NH}_{4}\right)_{2} \mathrm{MoS}_{4}$ was dissolved in water to form a $\mathrm{ca}$. $0.0045 \mathrm{M}$ solution before dispersing on an holey carbon E-chip (Protochips). Atomic resolution imaging was performed on a JEOL-JEMARM300CF equipped with probe and image aberration corrector, at $300 \mathrm{kV}$ in STEM mode.

The beam induced formation of two dimensional $\mathrm{MoS}_{2}$ from amorphous precursor shows the simultaneous formation of vertical stacks and horizontal layers before the eventual disappearance of the vertical stacks (Figure 1). HAADF images clearly show the verticality of the stacks based on the difference in intensity. The change in orientation of the $\mathrm{MoS}_{2}$ from vertical to horizontal follows previous works that indicate the preference for the horizontal position in order to reduce the total energy of the system. [2] However, the beam induced process has a clear preference for the horizontal orientation, bypassing any vertical stack formation in the majority of the illuminated area to directly form horizontal layers of $\mathrm{MoS}_{2}$. The contrast to traditional thermolysis indicates a potential difference in mechanism for the formation of the material.

To investigate the dynamics of the bonding changes in situ, power spectra were calculated from the atomic resolution images, similar to previous dynamics analysis. [3] The transformation was monitored and show the direct appearance of peaks associated with crystalline $\mathrm{MoS}_{2}$ with improving crystallinity as the peaks become sharper under continued beam irradiation (Figure 2). The formation of the two dimensional material seems to bypass the $\mathrm{MoS}_{3}$ intermediate traditionally thought to occur during thermolysis. By analyzing the bonding changes during this electron beam induced process, we are able to understand the stages of material transformation.

Observing a beam induced transformation at atomic resolution provides a more comprehensive understanding of the atomic movement during the transition from the amorphous precursor to two dimensional $\mathrm{MoS}_{2}$. The in situ observations show a different process that prioritizes the formation of horizontal $\mathrm{MoS}_{2}$ rather than forming primarily traditional vertical stacks before falling over. The process was analyzed based on the power spectra of the images, showing formation of the $\mathrm{MoS}_{2}$. Understanding this transformation process is important as a new different way to control the generation of $2 \mathrm{D} \mathrm{MoS} 2$. 

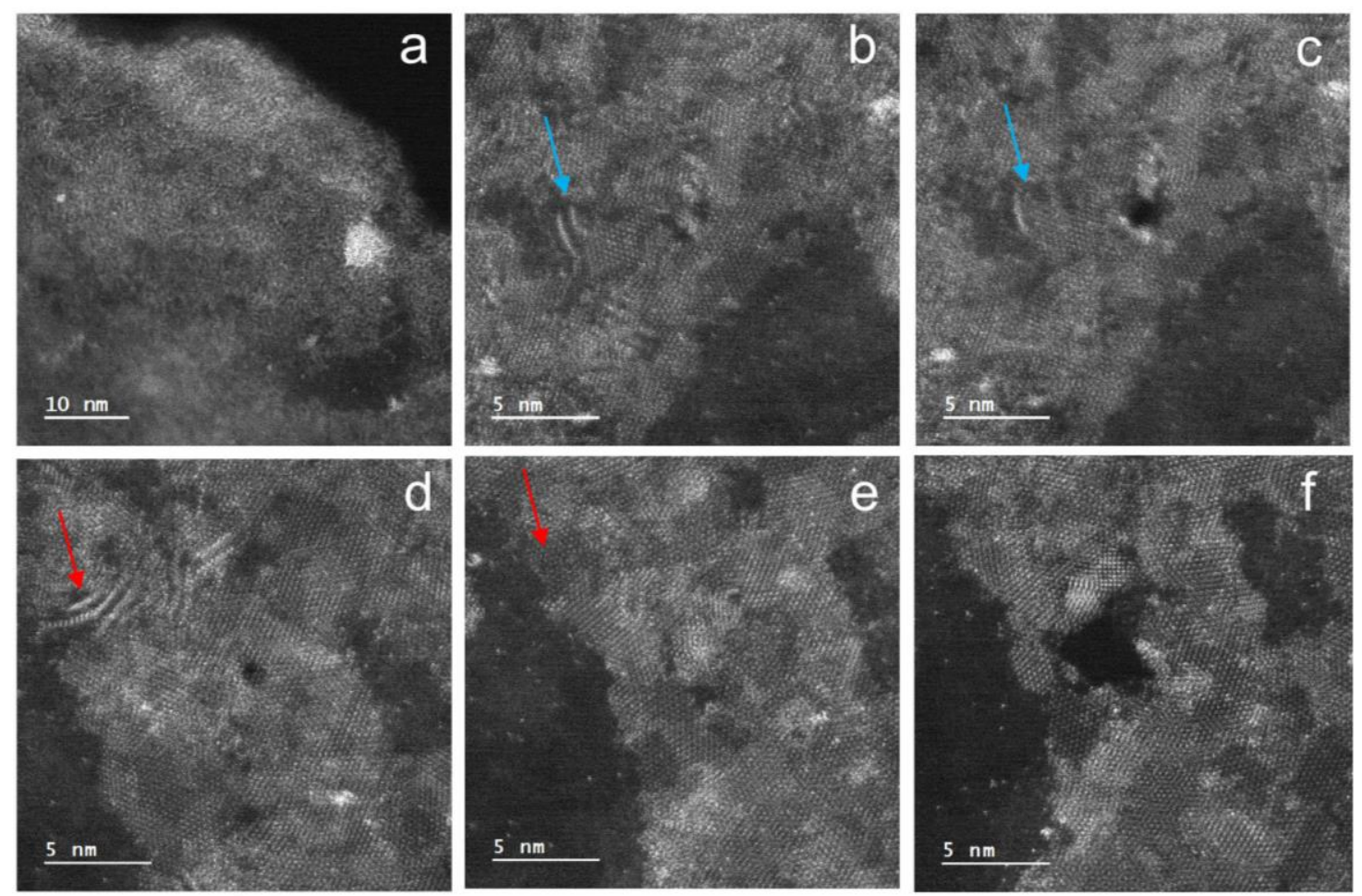

Figure 1. Sequential HAADF STEM images showing the formation of two dimensional MoS2 from amorphous precursor (a-f). The vertical stacks of MoS2 change orientation and contribute to growth of the horizontal layers (blue and red arrows).

a

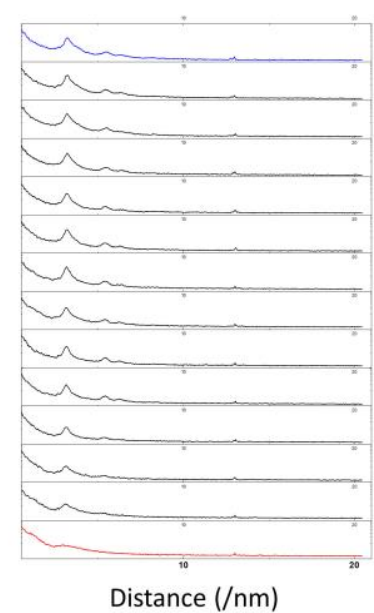

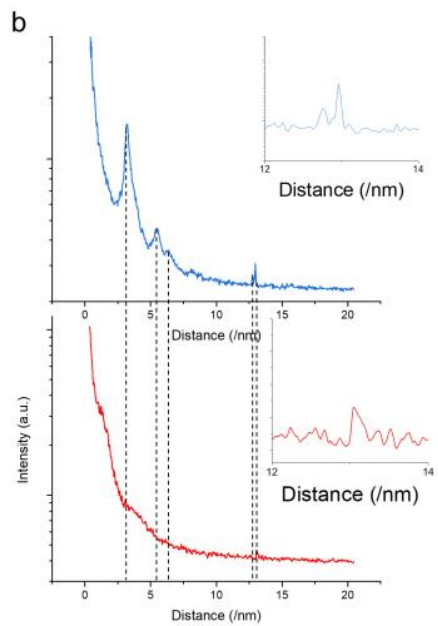

Figure 2. (a) Power spectra series of the atomic resolution STEM images for the transformation with (b) the first (red) and last (blue) image emphasized. Inset, magnification of the region between 12 and 14 nm1 . 


\section{References}

[1] R. Walton, et al. Chem. Mater. 10 (1998) p. 3737-3745

[2] L. Fei, et al. Nat. Comm. 7 (2016) 12206

[3] W. Gao, et al. Sci. Adv. 5 (2019) eaau9590 